\title{
Correction of a Class II Occlusion in an Adult
}

\author{
Fabio Savastano* \\ International College of Neuromuscular Orthodontics and Gnathology (ICNOG), Piazza Berlinguer 14, 17031 ALBENGA (SV), Italy
}

Received: August 17, 2014; Accepted: October 15, 2014; Published: November 05, 2014

*Corresponding author: Fabio Savastano, International College of Neuromuscular Orthodontics and Gnathology (ICNOG), Piazza Berlinguer 14,17031 ALBENGA (SV), Italy, Tel: +393-896-292-929; E-mail: savastano@ICNOG.com

\begin{abstract}
Introduction: Mandibular advancement and vertical correction with extrusion are two basic procedures that permit the skilled orthodontist to re-posture the mandible in Class II deep-bite occlusion with a disto-positioned mandible.

Aim: To verify the entity and the possibility of mandibular advancement in a non growing deep-bite patient while improving vertical dimension.

Methods: The diagnostic procedure is based on the use of (TENS) (Trans-cutaneous Electrical Nerve Stimulator) with application of the basic principles as described with the Neuromuscular Theory and technique. Treatment of a Class II occlusion on an adult patient without the use of mandibular tracking devices and without the aid of electromyography is described.

Results: Mandibular advancement and increase in vertical dimension were achieved. Flaring of lower incisors and head extension improved overall aesthetics.

Conclusions: The use of TENS is a valid diagnostic aid for predicting mandibular advancement in the adult patient.

Keywords: Neuromuscular orthodontics; Trans-cutaneous Electrical Nerve Stimulator; Mandibular posture; Deep-bite; Freeway space
\end{abstract}

\section{Introduction}

Treatment of class II occlusion in patients with vertical discrepancies has been an orthodontic issue clinicians have dwelt with for years. Vertical deficiency in Class II results in what orthodontists call deep-bite. Neuromuscular or Functional orthodontists would properly refer to them as "Cases with Excess Free-way Space". Attempt mandibular advancement or distalization of the upper arch, are only a few of the approaches orthodontists have chosen. Mandibular advancement is not advisable in the adult, as growth is no longer present and any repositioning of the condyle will likely not be accepted functionally and anatomically.

The Neuromuscular Theory [1,2] has opened a breach in the mechanical approach that has dominated orthodontics from its beginning. Doctors that have embraced a neuromuscular concept as the starting point for their diagnostics understand how, in certain adult patients, mandibular repositioning is indeed advisable. For those who are not familiar with the diagnostic procedure, a TENS appliance is used for determining a physiological cranio-mandibular relationship.

The patient's vertical dimension that includes inter-occlusal space or Freeway Space (FWS) is considered to be an adaptive physiologic parameter [2,3]. McNamara et al. 1978, [4] describe the rest position as determined by the activity of the fusimotor system of the elevator muscles via efferent input by the Central Nervous System (CNS) and inputs through stimuli from peripheral receptors located in the TMJ, gingiva, periodontal ligaments tongue and palate.

The TENS device stimulates the motor branches of the $\mathrm{V}^{\text {th }}$ and VII ${ }^{\text {th }}$ cranial nerves thus relaxing the mandibular postural muscles. Two electrodes are placed on the coronoid notch bilaterally and a third, neutral, is place at equal distance from the first two, behind the lower neck. The procedure requires placing an occlusal interference such as wax to avoid occlusal contacts during the pulsing session that lasts at least 45 minutes. This is done to avoid afferent peripheral stimuli to the CNS that could re-condition mandibular posture to adaptive functional, habitual, parameters. The newly obtained position of the mandible is then recorded with inter-occlusal bite recording material. This recording is then used for cast mounting. This procedure guarantees an immediate visual feedback of the possibility and quantity of mandibular anterior repositioning. Not all patients have a significant increase in freeway space and mandibular advancement after TENS application, however it does not come with surprise, that in Class II relationships (normo or deep-bite) we often see mandibular advancement and increase in freeway space after this procedure, $[5,6]$. Figure 1 a shows the new J5 Myomonitor that is the latest advancement in TENS therapy and diagnostic aid. This device can relax muscles, treat TMD dysfunction and pain, and increase mandibular range of motion and local blood flow. It is the only true simultaneous four channel bi-lateral stimulator of its kind.

We are all familiar to Functional Appliances and when to use them. The effort to develop a non-mechanical approach to solving orthodontic problems, led several orthodontists to develop 

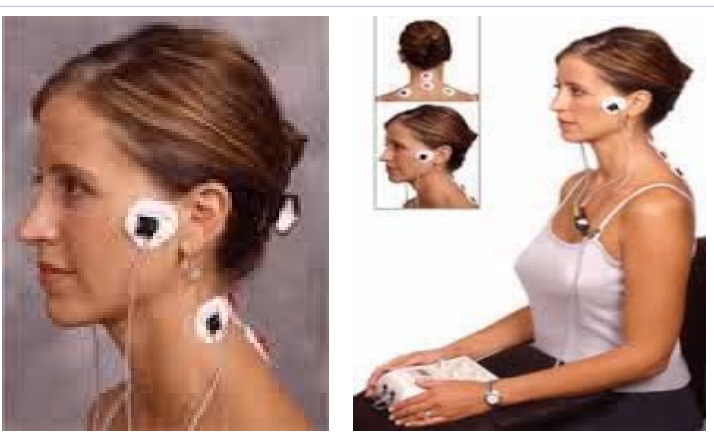

Figure 1a: Myomonitor J5 from Myotronics Inc, Kent, WA. USA.


Figure 1b: Pretreatment extra-oral photographs.
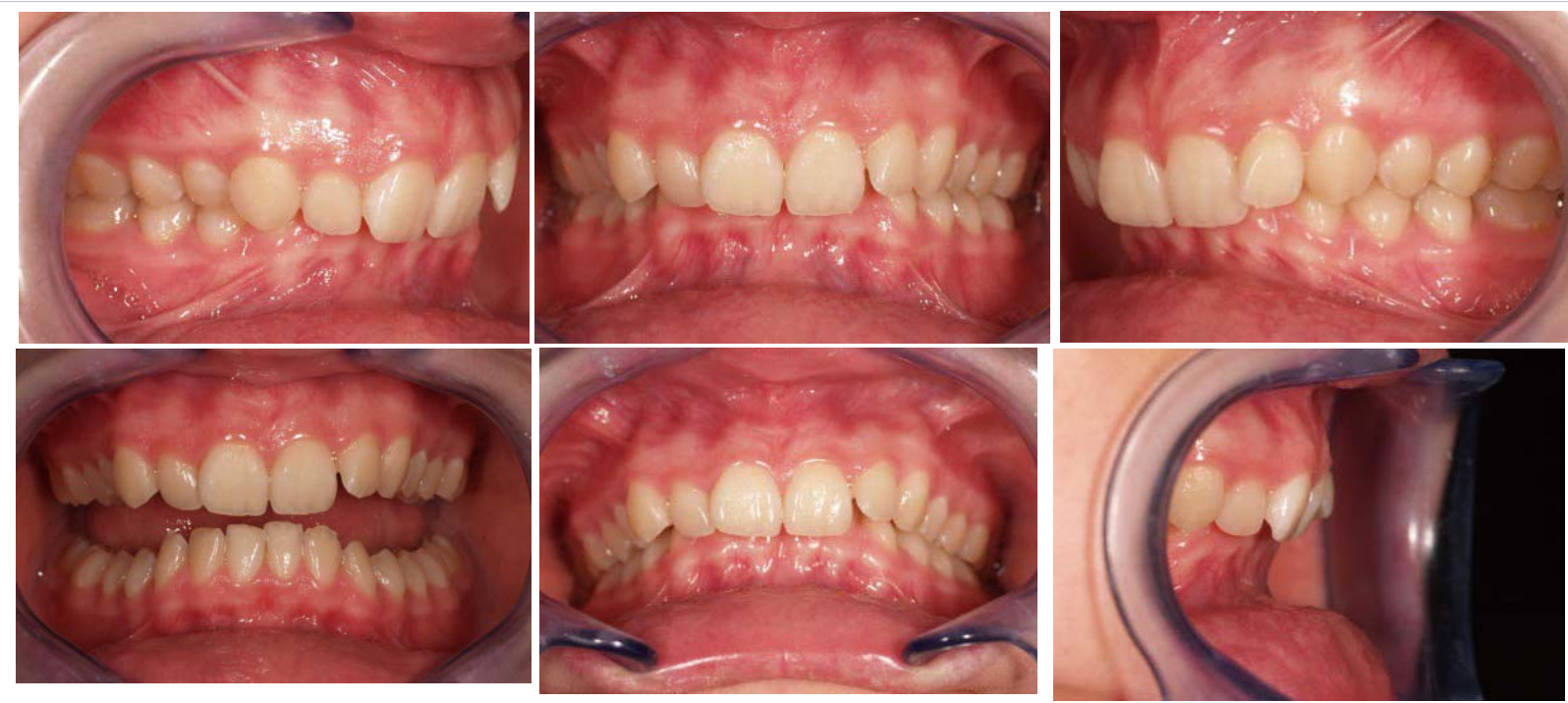

Figure 2: Pretreatment intraoral photographs. 


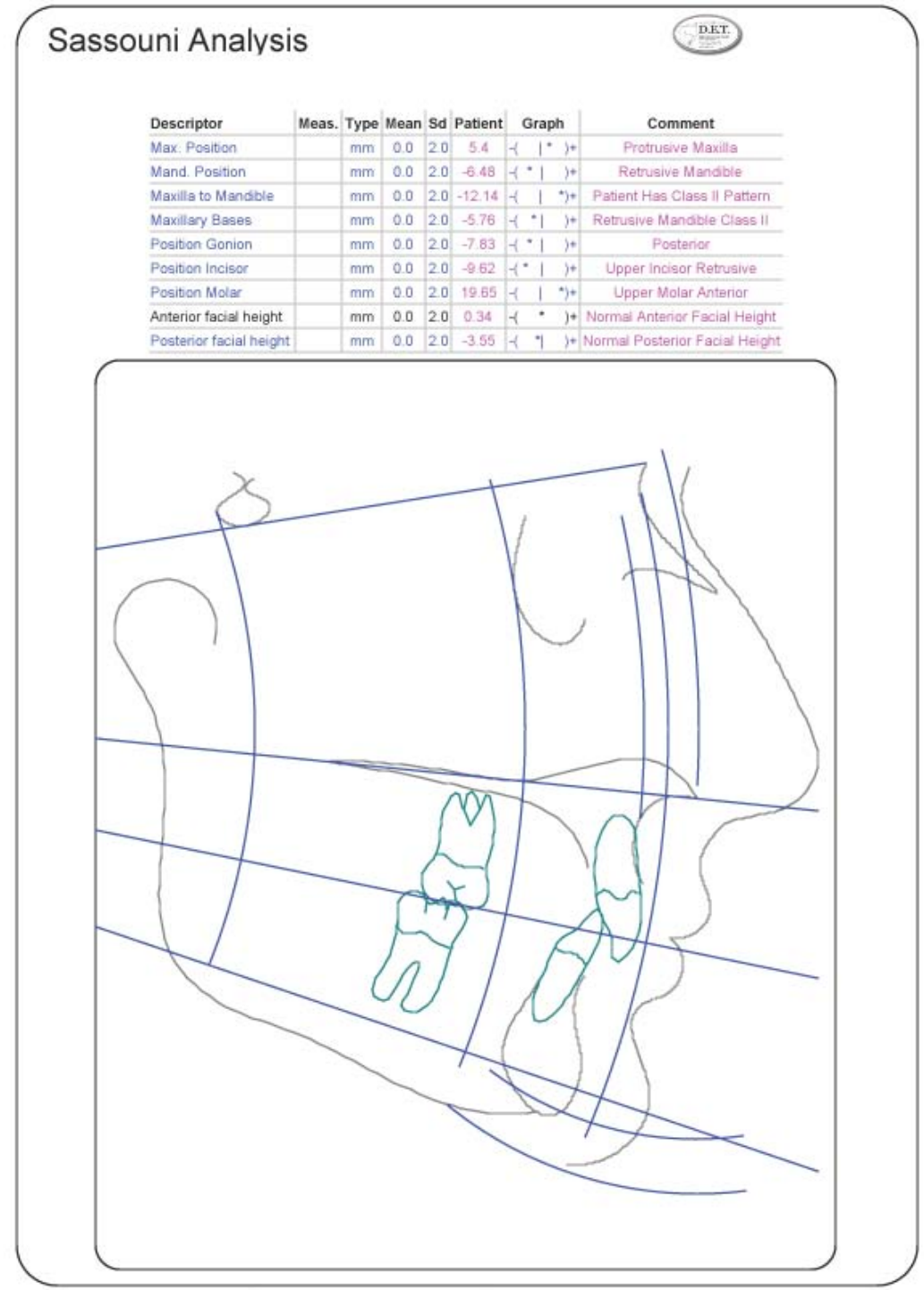

Figure 3: The Sassouni cephalometric analysis showed a Class II Pattern with a retrusive mandible.
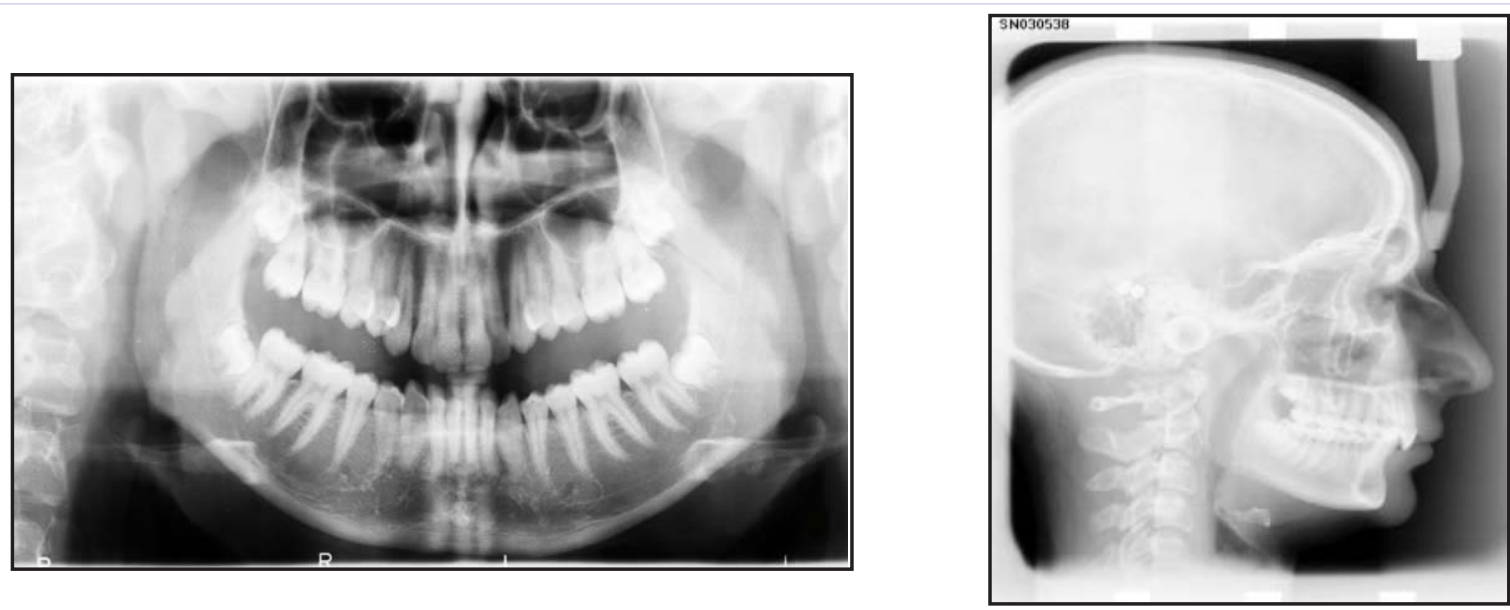

Figure 4: Pretreatment panoramic and cephalometric radiographs. 


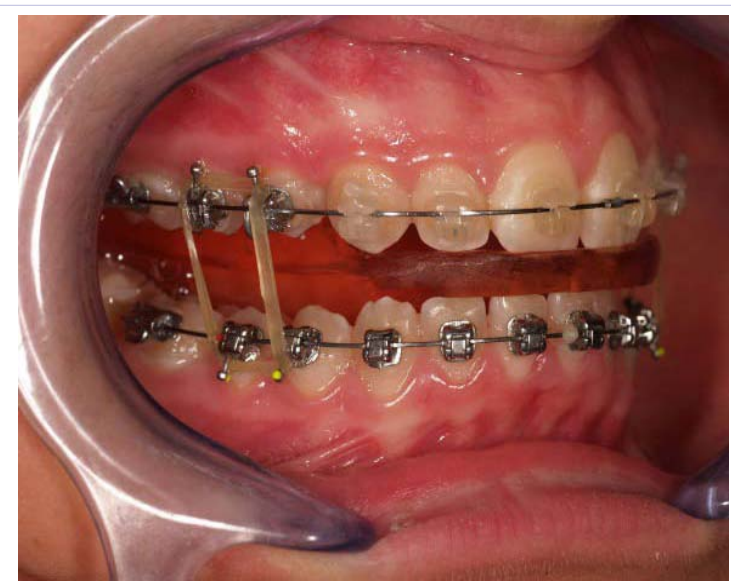

Figure 5: daywear of light elastics on both sides.

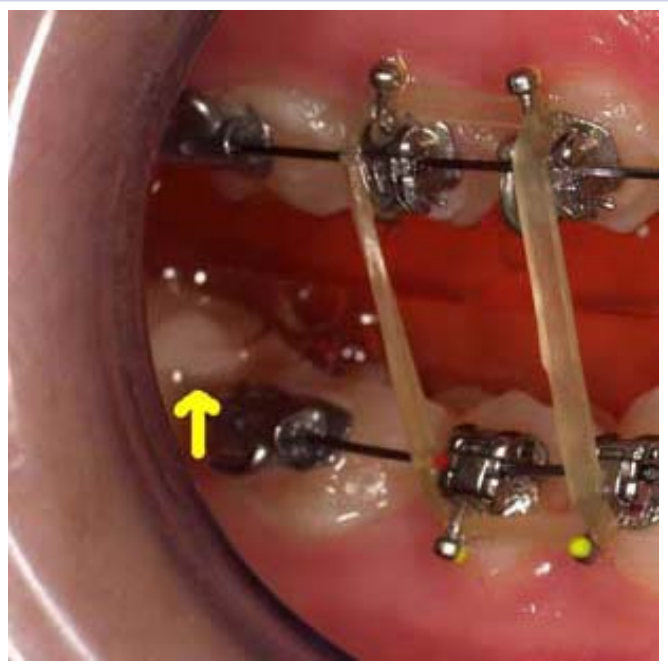

Figure 6: The elastics worn on the 24-hour basis, were for this reason, active also without the Functional Appliance.
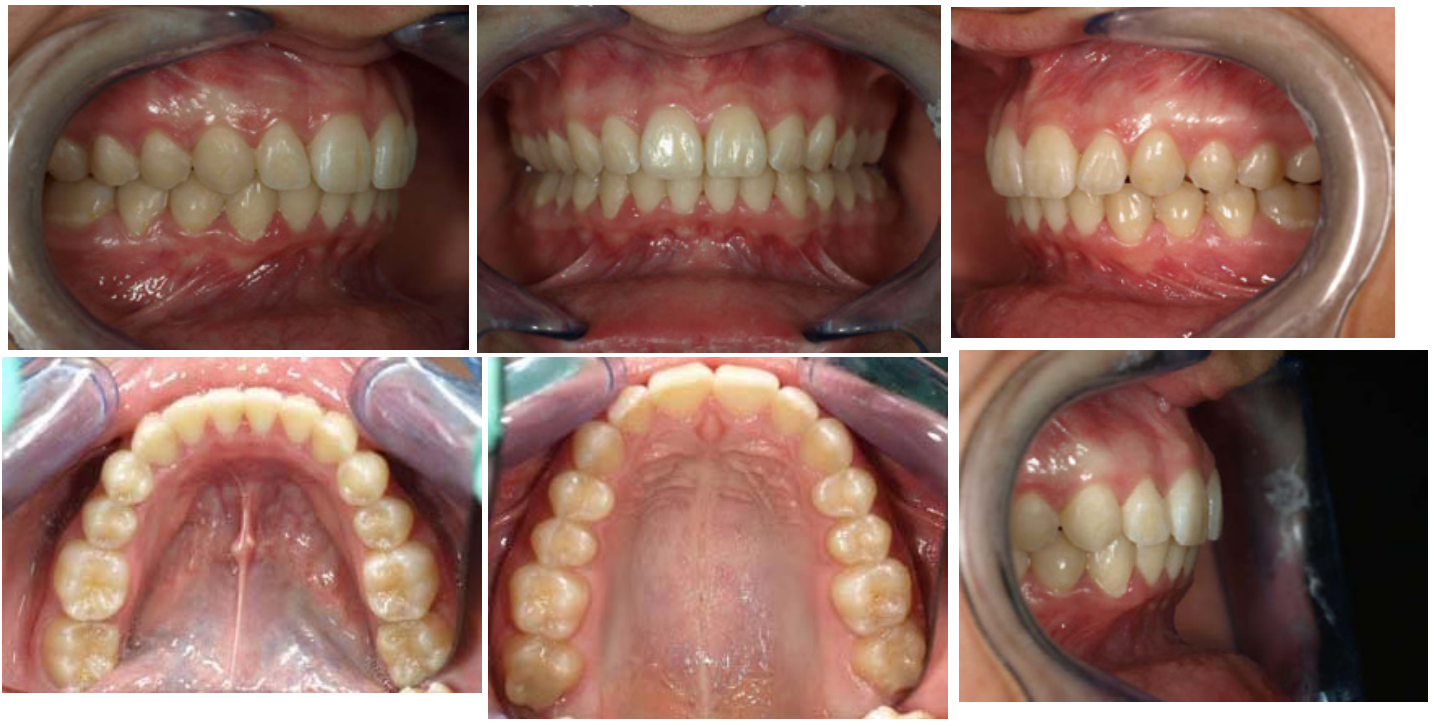

Figure 7: Post-treatment intraoral photographs. 

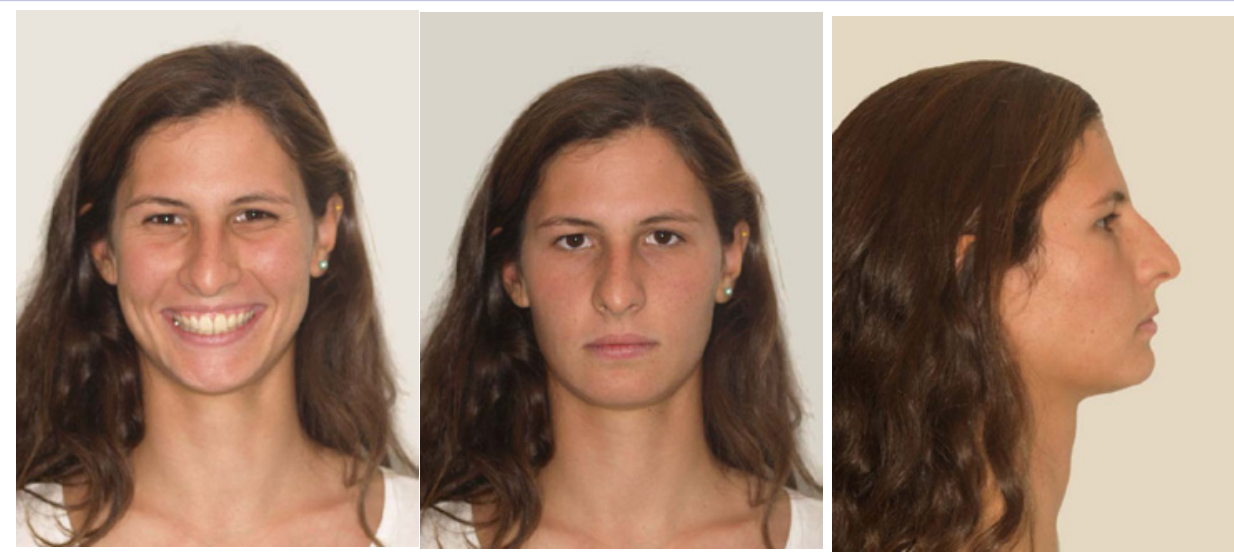

Figure 8a: Extraoral post-treatment photographs.
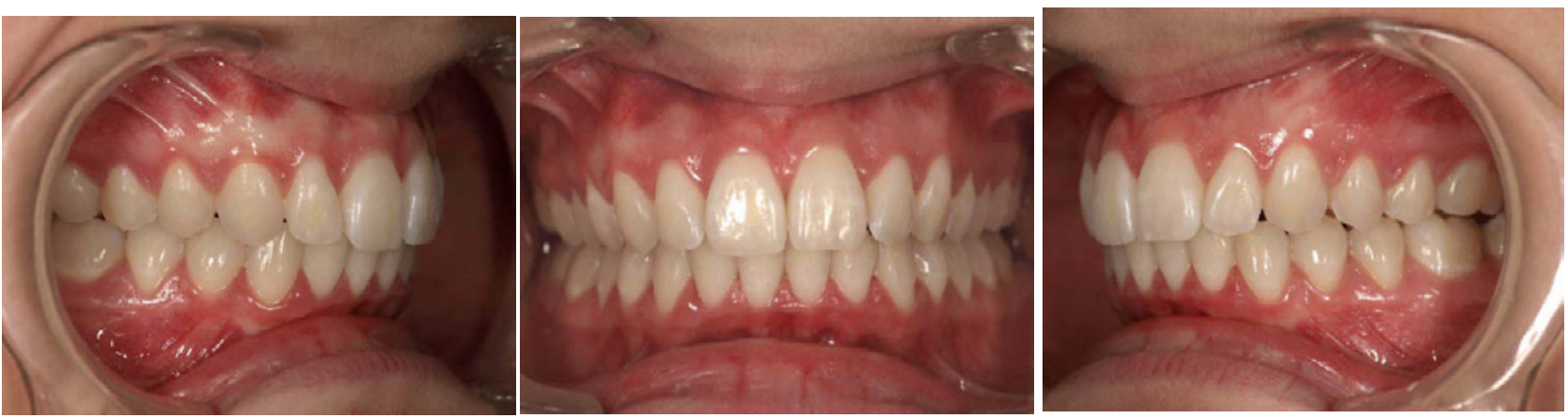

Figure 8b: Intraoral post-treatment photographs.

appliances that would in some way restore the function. Now that technology embraces our everyday life, we learn to bring these changes to our profession as well. Mandibular Tracking, Electromyography and other sophisticated procedures assure us a better understanding of a functional occlusion. Diagnosis and Etiology

A 16-year old female sought orthodontic treatment to my office for misaligned teeth and a gummy smile. She had a convex profile with a prominent nose (Figure1b).

The intraoral examination showed healthy periodontal tissues and a good oral hygiene. Deep-bite was evident as well as excessive curve of Speed of the lower arch. The upper incisors were inclined lingually. She exhibited a Class II malocclusion. Cervicalgia and spontaneous bilateral joint pain were often present (Figure 2).

\section{Treatment Objectives}

The treatment objectives for this patient were to correct the molar relationship and the deep bite. The correction of the deep bite was to be achieved via extrusion of the posterior segments as well as a slight intrusion of the upper incisors. Correction of the molar relationship was to be done via mandibular advancement. This was possible, as one of the main diagnostic steps was checking the re-posturing of the mandible after the application of a TENS during the diagnostic assessment. This patient showed a reasonable amount of advancement and enough freeway space to decide for a mandibular repositioning and extrusion of the posterior segments. An increase in facial profile aesthetics was desirable. Cephalometric radiographs and extra-oral photographs were taken with a Natural Head Posture technique using a mirror [7] (Figure 3,4).

\section{Treatment Alternatives}

The treatment alternatives included:

\section{No treatment}

2.Extraction of the upper first and lower first premolars

\section{Extraction of upper first premolars}

4.Extraction of lower third molars and upper third molars and mandibular surgical advancement

\section{Non extraction treatment}

The patient's main complaint was the misalignment of the upper incisors and a retrusive mandible. All treatment alternatives were refused based on the fact that patient had no intension on having extractions or surgery. Non-extraction treatment was therefore chosen, even though there was a concrete possibility of lower arch incisal flaring and increase of the naso-labial angle due to slight upper arch distalization. 


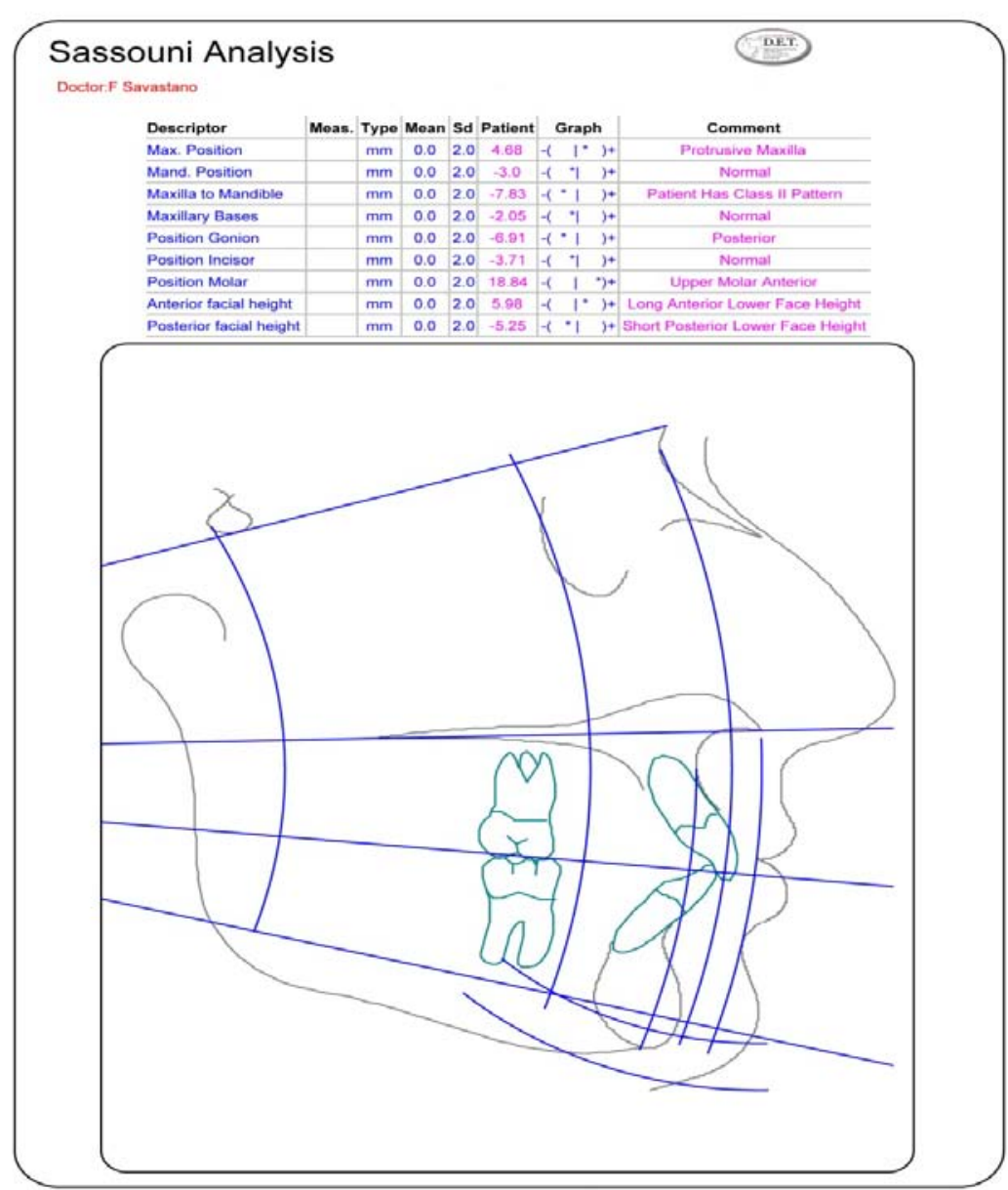

Figure 9a: Post-treatment Cephalometric analysis (D.E.T. WWW.CEPHANALYSIS.COM).

\section{Treatment Progress}

Treatment was started in January 2011 by bonding the upper arch only, from the first molars on each side. Initial alignment was achieved using a 0.014 nickel-titanium alloy archwire and subsequently a $0.017 \times 0.025$ Copper $\mathrm{Ni}-\mathrm{Ti}$.

As soon as the upper incisors were aligned, thus leaving enough space for bonding the lower arch, in June 2011, a Bionator was used to permit extrusion of the middle segments. This was helped with daywear of light elastics on both sides (Figure 5).

The Bionator was applied only while the patient was at home, including nightwear. Build-ups in composite were also done on the lower and upper second molars and were determined by the mandibular position at rest after the use of TENS as previously described. These build-ups would position the mandible in the desired posture during the day when not using the Bionator. The elastics worn on the 24-hour basis were for this reason, active also without the Functional Appliance (Figure 6). The extrusion with elastics lasted until January 2012. Final alignment was obtained by July 2013 when patient was set to retention with upper and lower removable appliances and instructed for night ware.

\section{Treatment Results}

Class I dental occlusion was obtained as well as a good profile change. Deep bite was resolved and alignment of both arches was achieved. Correction of the excess of the curve of Spee was also one of our objectives (Figures 7-11), Joint pain was also resolved (Tables 1,2,3).

The Grid Analysis is obtained by dropping a perpendicular line from the sella to the occlusal plane. Variations of the maxilla and the mandible can be measured in reference to the grid [8]. Note that the occlusal plane has been detected as indicated by Downs [9]. 


\begin{tabular}{|c|c|c|c|c|c|c|c|}
\hline Descriptor & Meas. & Type & Mean & Sd & Patient & Graph & Comment \\
\hline Max. Position & & $\mathrm{mm}$ & 0.0 & 2.0 & 5.4 & $-\left(I^{*}\right)$ & Protrusive Maxilla \\
\hline Mand. Position & & $\mathrm{mm}$ & 0.0 & 2.0 & -6.48 & $-\left(*^{*} \mid\right)$ & Retrusive Mandible \\
\hline Maxilla to Mandible & & $\mathrm{mm}$ & 0.0 & 2.0 & -12.14 & $-\left(\left.\quad\right|^{*}\right)+$ & Patient has class II Pattern \\
\hline Maxillary Bases & & $\mathrm{mm}$ & 0.0 & 2.0 & -5.76 & $\begin{array}{lll}-( & 1 & 1\end{array}$ & Retrusive Mandible Class II \\
\hline Position Gonion & & $\mathrm{mm}$ & 0.0 & 2.0 & -7.83 & $-(* \mid) \quad+$ & Posterior \\
\hline Position Incisor & & $\mathrm{mm}$ & 0.0 & 2.0 & -9.62 & $-\left(^{*} \quad \mid \quad 1\right)+$ & Upper Incisor Retrusive \\
\hline Position Molar & & $\mathrm{mm}$ & 0.0 & 2.0 & 19.65 & $-\left(||^{*}\right)+$ & Upper Molar Anterior \\
\hline Anterior facial height & & $\mathrm{mm}$ & 0.0 & 2.0 & 0.34 & $-(\quad *)+$ & Normal Anterior Facial Height \\
\hline Posterior facial height & & $\mathrm{mm}$ & 0.0 & 2.0 & -3.55 & $-\left({ }^{*} \mid 1\right)+$ & Normal Posterior Facial Height \\
\hline Descriptor & Meas. & Type & Mean & Sd & Patient & Graph & Comment \\
\hline Max. Position & & $\mathrm{mm}$ & 0.0 & 2.0 & 4.68 & \begin{tabular}{l|ll}
-( & $*$ & 1
\end{tabular} & Protrusive Maxilla \\
\hline Mand. Position & & $\mathrm{mm}$ & 0.0 & 2.0 & -3.0 & $-\left({ }^{*}\right)$ & Normal \\
\hline Maxilla to Mandible & & $\mathrm{mm}$ & 0.0 & 2.0 & -7.83 & -()$^{*} \mid$ & Patient has Class II Pattern \\
\hline Maxillary Bases & & $\mathrm{mm}$ & 0.0 & 2.0 & -2.05 & $-\left({ }^{*} \mid\right)+$ & Normal \\
\hline Position Gonion & & $\mathrm{mm}$ & 0.0 & 2.0 & -6.91 & $-\left(\begin{array}{lll}* & 1 & )+\end{array}\right.$ & Posterior \\
\hline Position Incisor & & $\mathrm{mm}$ & 0.0 & 2.0 & -3.71 & $-1 \quad * 1$ & Normal \\
\hline Position Molar & & $\mathrm{mm}$ & 0.0 & 2.0 & 18.84 & $\left.1{ }^{*}\right)+$ & Upper Molar Anterior \\
\hline Anterior facial height & & $\mathrm{mm}$ & 0.0 & 2.0 & 5.98 & $\begin{array}{llll}-( & 1 & * & 1\end{array}$ & Long Anterior Lower Face Height \\
\hline Posterior facial height & & $\mathrm{mm}$ & 0.0 & 2.0 & -5.25 & $-\left({ }^{*} \mid\right)+$ & Short Posterior Lower Face Height \\
\hline
\end{tabular}

Figure 9b: Sassouni comparison analysis table, before and after treatment. Mandibular position, incisor and lower face height improve.
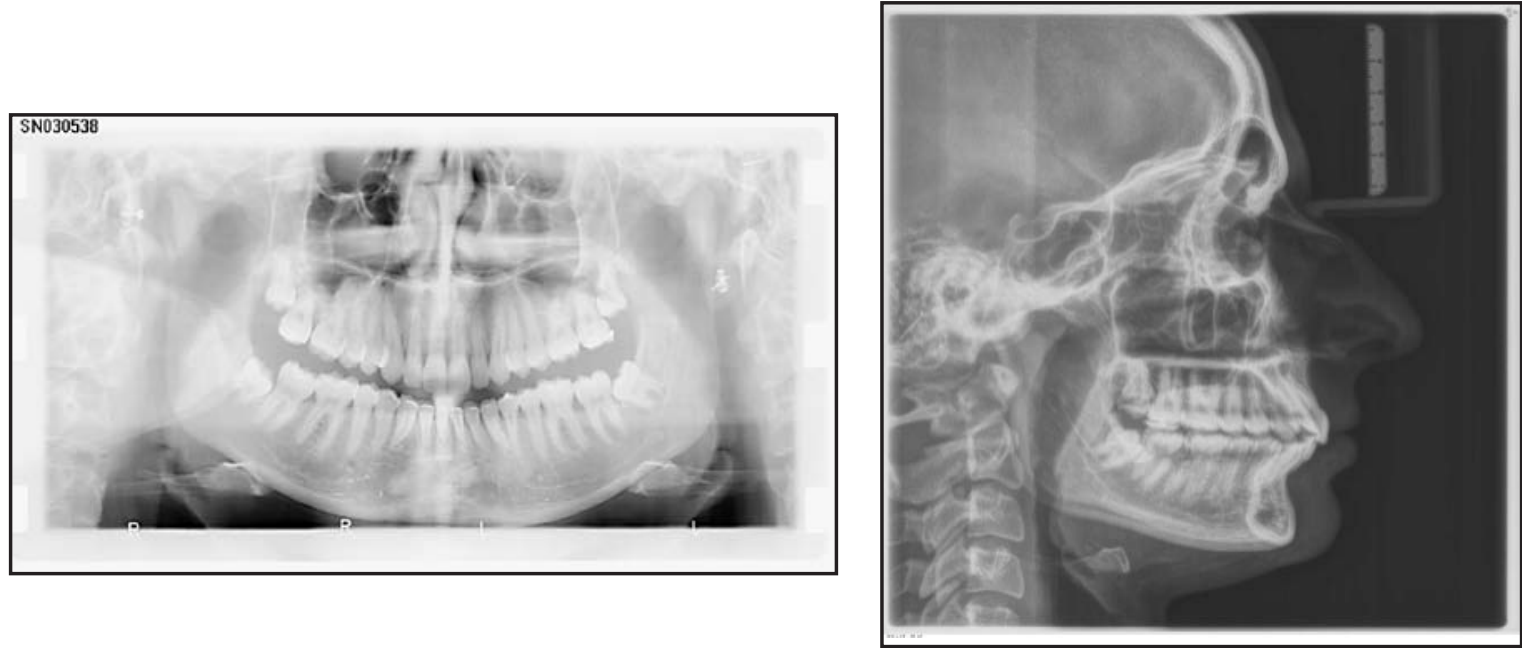

Figure 10a: Post-treatment radiographs.

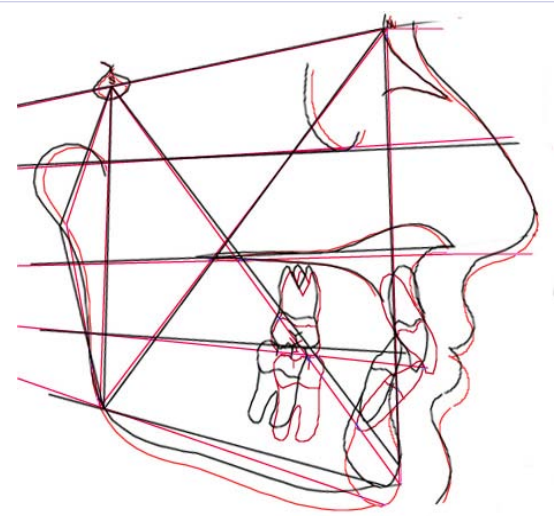

Figure 10b: Craniofacial superimpositions: 15 years 11 months (black) and 18 years 5 months (red). The plane of occlusion was opened without retrusion of the mandible. Since the opening movement of the mandible is a clock wise rotation, the increase in vertical dimension is due to teeth extrusion and mandibular advancement.. 
Table 1: Table showing before and after treatment results of (SNA) Sella-Nasion-Point A Angle, (SNB) Sella- Nasion-Point B Angle, (FMA) FrankfortMandibular Plane Angle, (IMPA) Incisor-Mandibular Plane Angle).

\begin{tabular}{|c|c|c|}
\hline Measurement & Before Treatment & After treatment \\
\hline SNA & 85.5 & 84.3 \\
\hline SNB & 79.2 & 79.2 \\
\hline FMA & 14.2 & 18.7 \\
\hline IMPA & 96.3 & 115.6 \\
\hline
\end{tabular}

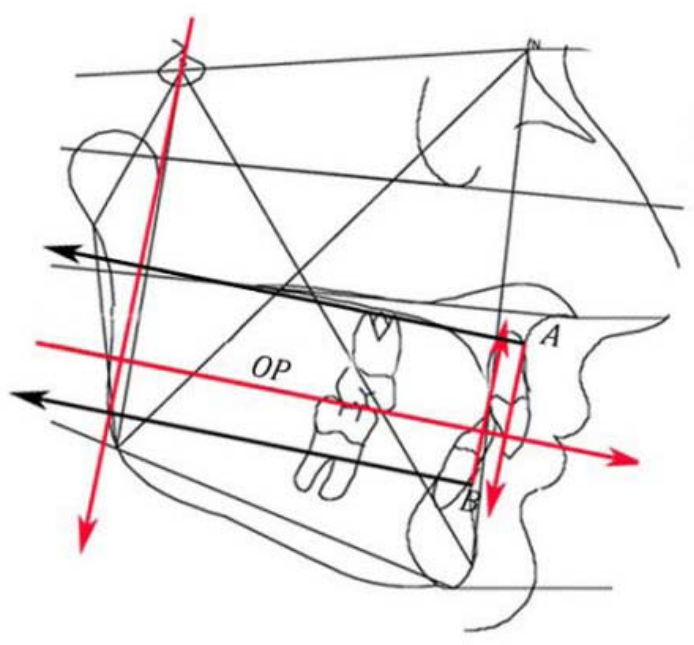

Figure 10c: Before treatment Grid Analysis.

Table 2: Point A; (B) Point B; (POP) Perpendicular to Occlusal plane from Sella.

\begin{tabular}{|l|c|}
\hline A to POP & $94.0 \mathrm{~mm}$ \\
\hline B to POP & $88.9 \mathrm{~mm}$ \\
\hline A to B on OP & $1.9 \mathrm{~mm}$ \\
\hline
\end{tabular}



Figure 10d: After treatment Grid Analysis.

Table 3: Values indicate that the treatment objectives were obtained.

\begin{tabular}{|c|c|}
\hline A to POP & $96.5 \mathrm{~mm}$ \\
\hline B to POP & $95.0 \mathrm{~mm}$ \\
\hline A to B on OP & $2.3 \mathrm{~mm}$ \\
\hline
\end{tabular}




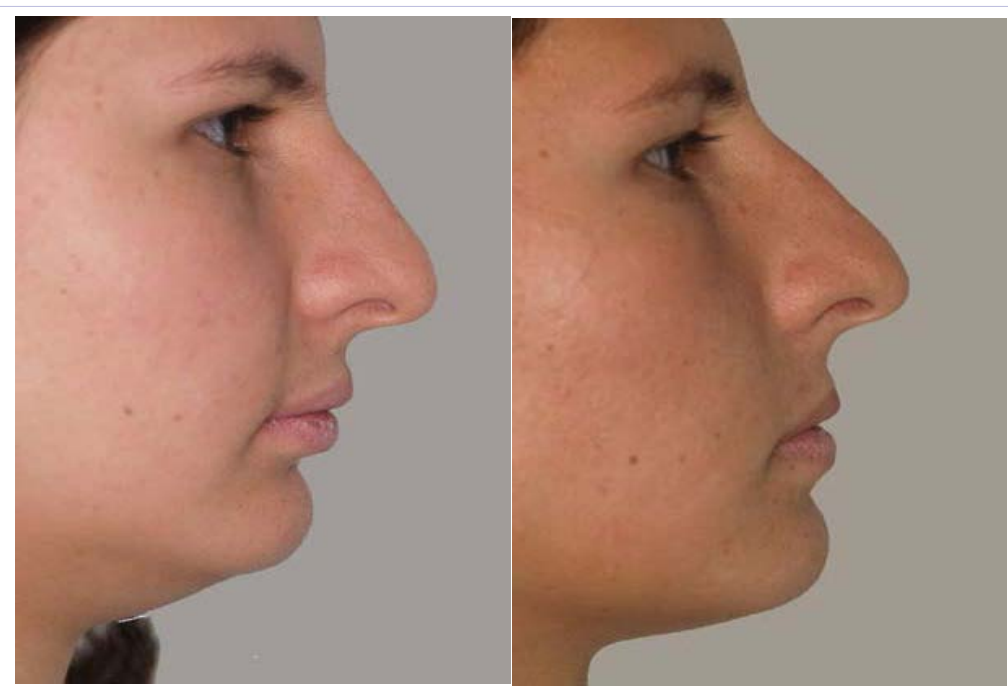

Figure 11: Pre and post-treatment profile photographs, natural head posture.

\section{Discussion}

Total treatment time was 30 months. Improving profile in Class II patients when starting treatment at 16 years of age is a challenge. Obviously, if we had started treatment during the growth spurt, we would have unlocked and freed the mandible in a much easier way. Improvement of facial profile would have been a logical consequence of upper maxillary expansion. Joint tenderness would have not been present; a clear indication of a distalized mandible.

\section{Cephalometrics}

The Sassouni analysis clearly shows improvement in mandibular advancement. This can be evaluated also from the Maxillary Basis results (Figures 3,9,10a). Superimposition shows lowering of the lip line in respect of the upper incisor and increase in lower vertical dimension. There are no dramatic changes in Sella Nasion A (SNA) point angle and Sella Nasion B (SNB) point angle as expected, while FMA shows an increase in vertical opening of the bite. IMPA has increased due to increase of mandibular incisor tipping (Figure 10b). Normal values for FMA are 20-30 and for IMPA between 76 and 99 as indicated by Tweed [10]. The Grid Analysis shows improvement in all the values indicated. This is due to the fact that this method is based on treatment results in respect of the mandibular plane (Figure $10 \mathrm{c}$ and $10 \mathrm{~d}$ ). If mandibular advancement had not occurred, the sole bite opening would have resulted in lower values of SNB angle.

\section{Results}

Lower facial height improved and Class I molar relationship was achieved. Joint pain was resolved during the functional appliance phase and vertical extrusion of the middle segments. Overall aesthetics improve dramatically due to orthodontic treatment and consequential head extension.
The treatment choices do vary from orthodontist to orthodontist. Any extractions in upper and/or lower arch would certainly not have allowed opening of the bite or any repositioning of the mandible. Any upper distalization would not have favored repositioning of the mandible in a more forward and lower position. Note that the nasolabial angle is more than acceptable at end of treatment (Figure 11).

Postural changes do occur, as head extension plays a dramatic role in improving the overall aesthetics of the patient. We can translate this in millimeters: a bite opening of a few millimeters is sufficient to determine extension of the head enough to be well noted from the orthodontist and his patients [11-13]. The result is generally a positive surprise for our patients and should be accounted for when planning treatment.

Labial tipping of the lower incisors is a small price that we must pay if we want to correct cases like these. Consider though that there is a good and large bone support for counter-clockwise growing mandibles and that the range of tolerance is in these cases augmented. Erdinc et al.'s [14] findings show that there is no statistical increase in lower arch relapse of lower anterior incisal crowding between extraction and non-extraction groups after orthodontic alignment $[14,15]$. In open-bite cases in which bone is thin and thus support of the incisors critical (Bjork), tolerance is reduced and torqueing lower incisors is a risky maneuver.

\section{Conclusions}

The use of TENS to determine mandibular rest position is not a new technique. Although there are several other ways to determine rest position, this case is one of the many that support the use of TENS for orthodontic purposes.

\section{References}

1. Neuromuscular dental diagnosis and treatment: Robert R. Jankelson Ishiyaku EuroAmerica, St. Louis: 1990. 687 pages, 1132 illustrations. American journal of orthodontics and dentofacial. 1991; 99(3): 283284. DOI: http://dx.doi.org/10.1016/S0889-5406(05)80267-7. 
2. Mohl ND. Neuromuscular mechanisms in mandibular function. Dent Clin North Am. 1978; 22(1): 63-71.

3. Niswonger ME. The rest position of the mandible and the centric relation. J.A.D.A., 1934. 21: p. 1572-1582.

4. Mc Namara JA, Carlson DS. Muskuloskeletal adaptation following orthognatic surgery in Craniofacial growth series. 1978, Needham Press: University of Michigan, Ann Aror, 1978 U.S.A.

5. Konchak PA, Thomas NR, Lanigan DT, Devon RM. Freeway space measurement using mandibular kinesiograph and EMG before and after TENS. Angle Orthod. 1988; 58(4): 343-50.

6. Monaco A, Cattaneo R, Marci MC, Marzo G, Gatto R, Giannoni M Neuromuscular diagnosis in orthodontics: effects of TENS on maxillomandibular relationship. Eur J Paediatr Dent. 2007; 8(3): 143-8.

7. Cooke MS, Wei SH. The reproducibility of natural head posture: A methodological study. Am J Orthod Dentofacial Orthop. 1988; 93(4): 280-8.

8. Wieslander L. Long-term effect of treatment with the headgear-Herbst appliance in the early mixed dentition. Stability or relapse? Am J Orthod Dentofacial Orthop. 1993; 104(4): 319-29.
9. Downs WB. Variations in facial relationships: Their significance in treatment and prognosis. Am J Orthod. 1948; 34(10): 812-40.

10. Tweed CH. The Frankfort-mandibular plane angle in orthodontic diagnosis, classification, treatment planning, and prognosis. Am J Orthod Oral Surg. 1946; 32: 175-230.

11.Vig PS, Showfety KJ, Phillips C. Experimental manipulation of head posture. Am J Orthod. 1980; 77(3): 258-68.

12. Solow B, Tallgren A. [Head position and craniofacial morphology]. Mondo Ortod. 1977; 19(6): 75-99.

13. Solow B, Tallgren A. Head posture and craniofacial morphology. Am J Phys Anthropol. 1976; 44(3): 417-35.

14. Erdinc AE, Nanda RS, Işiksal E. Relapse of anterior crowding in patients treated with extraction and nonextraction of premolars. Am J Orthod Dentofacial Orthop. 2006; 129(6): 775-84.

15. Rossouw PE, Preston CB, Lombard C. A longitudinal evaluation of extraction versus nonextraction treatment with special reference to the posttreatment irregularity of the lower incisors. Semin Orthod. 1999; 5(3): 160-70. 